MODELING, IDENTIFICATION AND CONTROL, 1999, VOL. 20, NO. 3, 177-187

doi:10.4173/mic.1999.3.3

\title{
Nonlinear control of ships minimizing the position tracking errors
}

\author{
SVEIN P. BERGE ${ }^{\prime}{ }^{1}$ KOHEI OHTSU $\ddagger$ and THOR I. FOSSEN $\dagger$
}

Keywords: Nonlinear control, Lyapunov stability, ship control, marine systems

In this paper, a nonlinear tracking controller with integral action for ships is presented. The controller is based on state feedback linearization. Exponential convergence of the vessel-fixed position and velocity errors are proven by using Lyapunov stability theory. Since we only have two control devices, a rudder and a propeller, we choose to control the longship and the sideship position errors to zero while the heading is stabilized indirectly. A Virtual Reference Point (VRP) is defined at the bow or ahead of the ship. The VRP is used for tracking control. It is shown that the distance from the center of rotation to the VRP will influence on the stability of the zero dynamics. By selecting the $V R P$ at the bow or even ahead of the bow, the damping in yaw can be increased and the zero dynamics is stabilized. Hence, the heading angle will be less sensitive to wind, currents and waves. The control law is simulated by using a nonlinear model of the Japanese training ship Shiojimaru with excellent results. Wind forces are added to demonstrate the robustness and performance of the integral controller.

\section{Introduction}

Conventional autopilots do not control the ships position directly, but indirectly via the ship's heading. The autopilot uses an external heading reference as input either from a heading set-point or from an external reference, e.g., electronic chart system (Fossen 1994). If there are external disturbances due to wind, currents and waves, the ship's position will not follow the desired track. To circumvent this problem, Godhavn (1997) proposed a control law where the ship's position (latitude and longitude) was controlled instead of the heading. One advantage by using position control instead of heading control is that the ship position will follow the desired track even if there are environmental disturbances. The desired track can be planned in advance or being re-planned during the voyage. The control law requires two control devices, since both the longitude and the latitude are controlled simultaneously. However, the control law will influence on the motions in all 3 degrees of freedom (surge, sway and yaw) since this is a MIMO-system with 2 inputs, 2 outputs and 3 states.

Since the heading loop is open, the ship's heading angle will not necessarily be tangential to the desired track. In fact the ship may rotate $180^{\circ}$. The ship can be compared to a trolley in the supermarket. Pulling the trolley from a point in the front is not a problem, since it will not rotate around. If you push it at a point at the handle towards a way-point, it will rotate. Similarly, for a ship, the resulting heading angle will

$\dagger$ Department of Engineering Cybernetics, Norwegian University of Science and Technology, N-7034 Trondheim, Norway. e-mail: spb,tif@itk.ntnu.no

\$Tokyo University of Mercantile Marine, 2-1-6 Etchujima, Koto-ku, Tokyo, Japan 135. e-mail: ohtsu@ipc.tosho-u.ac.jp

'Partially supported by the Norwegian Research Council and Kværner ASA.

This paper is a slight revision of a paper presented at IFAC International Conference on Control Applications in Marine Systems, CAMS'98, 27-30 October 1998, Fukuoka, Japan. 
depend on the choice of the VRP and the external disturbances from wind, currents and waves. The VRP is defined as a point inboard or even in front of the ship which is supposed to track the desired path. The VRP was first introduced by Lindegaard (1997).

In this framework, the position tracking error is defined as the position error between the VRP and the desired position given by a smooth trajectory decomposed into the vessel-fixed frame. The position tracking errors correspond to the deviations in the longship and the transverse directions (cross-track error). The nonlinear control law, which forces the tracking errors to zero, is derived by using feedback linearization. The control law has a simpler structure than the nonlinear control laws derived by Fossen et al. (1998) and Godhavn et al. (1998) where vectorial backstepping were applied. Both the position and the velocity tracking errors converge to zero exponentially. Further, the zero dynamics is analyzed and stability is proven by using Lyapunov stability theory. The zero dynamics will depend on the forward speed and the selected VRP. By increasing the distance to the $V R P$, the closed-loop system will be less sensitive for external disturbances. The main difference of our approach and the linear controller of Holzhüter and Schultze (1996) is that nonlinear theory is used to prove GES.

The surge velocity, sway velocity and yaw rate are assumed measured and the earth-fixed position is assumed measured by a DGPS receiver. If velocity measurements are not available, a nonlinear observer can be used, see Fossen and Strand (1999).

The main contribution in this paper is that the proposed control law guarantees zero position and velocity error along the desired trajectory even with a constant unknown disturbance. The zero dynamics is proven also to be stable if the VRP is chosen ahead of the center of the rotation. Further, this controller has the same structure as a PID controller and the controller gains for the longship and transverse direction can be selected independently.

\section{Tracking Control Law}

\subsection{Vessel-fixed position tracking error}

As seen from Figure 1, both $e_{1}$ and $e_{2}$ are functions of $\psi$. It is convenient to decompose the position error into the vessel-fixed coordinate system rather than using the earth-fixed system as a reference frame. The main motivation for this is to have

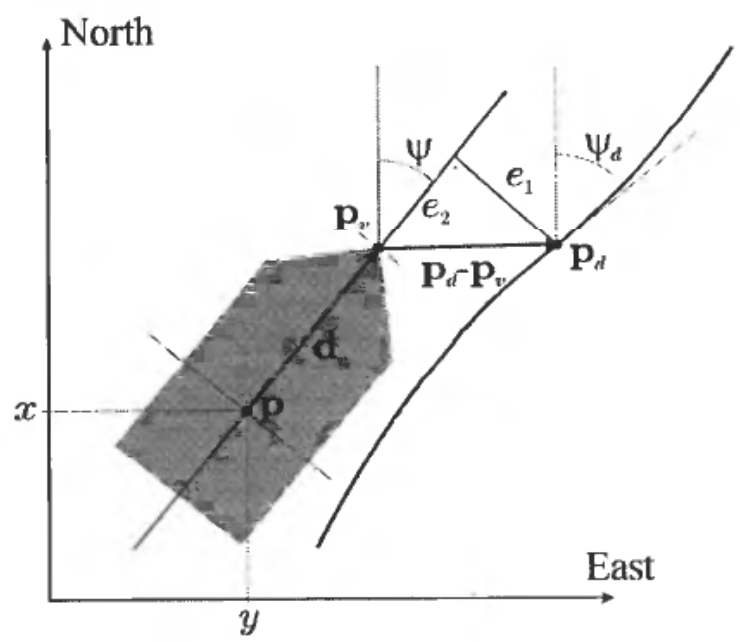

Figure 1. Definition of vessel-fixed position errors. 
different gains for the longship and transverse motions and to avoid heading dependent gains. The position tracking error $\mathbf{e}=\left[e_{1}, e_{2}\right]^{T}$ can be written as

$$
\mathbf{e}=\mathbf{R}^{T}(\psi)\left(\mathbf{p}_{d}-\mathbf{p}_{v}\right)
$$

where the transformation matrix $\mathbf{R}$ and the kinematic equations are given by

$$
\begin{aligned}
\mathbf{R}=\mathbf{R}(\psi) & =\left[\begin{array}{cr}
\cos \psi & \sin \psi \\
\sin \psi & \cos \psi
\end{array}\right] \\
\mathbf{R R}^{T} & =\mathbf{I}_{2 \times 2} \\
\dot{\mathbf{p}} & =\mathbf{R}(\psi) \mathbf{v} \\
\dot{\psi} & =r
\end{aligned}
$$

Here $\mathbf{v}=[u, v]^{T}$ and $\mathbf{p}=[x, y]^{T} . u$ is the alongship velocity (surge), $v$ is the athwarthship velocity (sway), $r$ is the rotational velocity (yaw) and $\psi$ is the heading angle. $\mathbf{p}_{d}=\left[x_{d}, y_{d}\right]^{T}$ is the desired position and $\mathbf{p}_{v}$ is the position of the VRP. Note, that

$$
\mathbf{p}_{v}=\mathbf{p}+\mathbf{R}(\psi) \mathbf{d}_{v}
$$

where $\mathbf{p}=[x, y]^{T}$ is the actual position referred to the center of rotation and $\mathbf{d}_{v}=\left[x_{v}, y_{v}\right]^{T}$ is the vector from the center of the rotation to the $V R P$ decomposed in the vessel-fixed frame. Hence:

$$
\begin{aligned}
\dot{\mathbf{e}} & =\dot{\mathbf{R}}^{T}(\psi)\left(\mathbf{p}_{d}-\mathbf{p}_{v}\right)+\mathbf{R}^{T}(\psi)\left(\dot{\mathbf{p}}_{d}-\dot{\mathbf{p}}_{v}\right) \\
& =r \mathbf{S e}+\mathbf{R}^{\mathrm{T}}(\psi) \mathbf{R}\left(\psi_{d}\right) \mathbf{v}_{d}-\mathbf{v}-\mathbf{R}^{\mathrm{T}}(\psi) \dot{\mathbf{R}}(\psi) \mathbf{d}_{v} \\
& =r \mathbf{S e}+\tilde{\mathbf{R}} \mathbf{v}_{d}-\left(\mathbf{v}-r \mathbf{S} \mathbf{d}_{v}\right) \\
& =r \mathbf{S e}+\tilde{\mathbf{R}} \mathbf{v}_{d}-\mathbf{T} \boldsymbol{v} \\
& =r \mathbf{S e}+\tilde{\mathbf{v}}
\end{aligned}
$$

where $\tilde{\mathbf{R}}=\mathbf{R}^{\mathbf{T}}(\psi) \mathbf{R}\left(\psi_{d}\right)=\mathbf{R}^{T}\left(\psi-\psi_{d}\right), \boldsymbol{\nu}=[u, v, r]^{T}$ and $\tilde{\mathbf{v}}=\tilde{\mathbf{R}} \mathbf{v}_{d}-\mathbf{T} \boldsymbol{\nu}$. Moreover:

$$
\begin{aligned}
\mathbf{v}_{d} & =\mathbf{R}^{T}\left(\psi_{d}\right) \dot{\mathbf{p}}_{d} \\
\dot{\mathbf{R}}^{T}(\psi) \mathbf{R}(\psi) & =r \mathbf{S} \\
\mathbf{S} & =-\mathbf{S}^{T}=\left[\begin{array}{rr}
0 & 1 \\
-1 & 0
\end{array}\right] \\
\mathbf{T} & =\left[\begin{array}{rrr}
1 & 0 & -y_{v} \\
0 & 1 & x_{v}
\end{array}\right]
\end{aligned}
$$

where $\psi_{d}$ is the desired leading angle computed from the reference generator, that is $\eta_{d}=\left[\mathbf{p}_{d}^{T}, \psi_{d}\right]^{T}$.

\subsection{Tracking Control Law}

In this section we derive a control law based on state feedback linearization. Integral action is added and the equilibrium point is proven to be globally exponentially stable (GES) using Lyapunov stability theory and the method of Megretski and Rantzer (1997). 
Proposition 1. The yaw rate $r(\mathrm{t})$ is bounded.

Proof. As shown in Appendix B, the zero dynamics only depends on the selected VRP and not on the controller gains. Since the zero dynamics is stable, $r(t)$ will be bounded.

The ship dynamics can be written as

$$
\dot{\boldsymbol{\nu}}=\mathbf{M}^{-1}(\boldsymbol{\tau}-\mathbf{n}(\boldsymbol{\nu})+\mathbf{b})
$$

where $\boldsymbol{r}$ is the thrust force in surge, sway and yaw and $\mathbf{b}$ is an unknown bias which is assumed to be constant. With two controls, a rudder and a propeller, the thrust force can be modeled as

$$
\boldsymbol{\tau}=\left[\begin{array}{cc}
b_{1} & 0 \\
0 & b_{2} \\
0 & t b_{2}
\end{array}\right]\left[\begin{array}{l}
u_{1} \\
u_{2}
\end{array}\right]=\mathbf{B u}
$$

where $b_{1}$ and $b_{2}$ are thrust force coefficients for the controllable pitch propeller (CPP) and the rudder, respectively, and $t$ is the distance from the center of rotation to the rudder. Let

$$
\begin{aligned}
& \dot{\tilde{\mathbf{v}}}=-\mathbf{K}_{D} \tilde{\mathbf{v}}-\mathbf{K}_{P} \mathbf{e}-\mathbf{K}_{I} \mathbf{z}-\mathbf{T} \mathbf{M}^{-1} \mathbf{b} \\
& \dot{\mathbf{z}}=\mathbf{e} \\
& \dot{\mathbf{e}}=r \mathbf{S e}+\tilde{\mathbf{v}}
\end{aligned}
$$

where $\mathbf{K}_{D}, \mathbf{K}_{P}, \mathbf{K}_{I}>\mathbf{0}$ and $\mathbf{z}$ is the integrated tracking error. The input $\mathbf{u}$ is found from

$$
\begin{aligned}
& \mathbf{T} \dot{\boldsymbol{\nu}}=\dot{\tilde{\mathbf{R}}} \mathbf{v}_{d}+\tilde{\mathbf{R}} \dot{\boldsymbol{v}}_{d}+\mathbf{K}_{D} \tilde{\mathbf{v}}+\mathbf{K}_{P} \mathbf{e}+\mathbf{K}_{I} \mathbf{z}+\mathbf{T} \mathbf{M}^{-1} \mathbf{b} \\
& \mathbf{T} \dot{\boldsymbol{\nu}}=\mathbf{T} \mathbf{M}^{-1}(\mathbf{B u}-\mathbf{n}(\boldsymbol{\nu})+\mathbf{b})
\end{aligned}
$$

Defining

$$
\begin{aligned}
& \mathbf{a}=\dot{\tilde{\mathbf{R}}} \mathbf{v}_{d}+\tilde{\mathbf{R}} \dot{\mathbf{v}}_{d}+\mathbf{K}_{D} \tilde{\mathbf{v}}+\mathbf{K}_{P} \mathbf{e}+\mathbf{K}_{l} \mathbf{z} \\
& \mathbf{a}=\mathbf{T} \dot{\boldsymbol{\nu}}-\mathbf{T} \mathbf{M}^{-1} \mathbf{b}
\end{aligned}
$$

yields

$$
\mathbf{u}=\mathbf{B}_{u}^{-1}\left[\mathbf{a}+\mathbf{T} \mathbf{M}^{-1} \mathbf{n}(\boldsymbol{\nu})\right]
$$

where $\mathbf{B}_{u}=\mathbf{T} \mathbf{M}^{-1} \mathbf{B}$ is invertible and $\mathbf{a}$ is the commanded acceleration. Define $\mathbf{x}=\left[\tilde{\mathbf{v}}^{T}, \mathbf{e}^{T}, \tilde{\mathbf{z}}^{T}\right]^{T}$ where $\tilde{\mathbf{z}}=\mathbf{z}+\mathbf{K}_{l}^{-1} \mathbf{T} \mathbf{M}^{-1} \mathbf{b}$, then the error dynamics becomes

$$
\dot{\mathbf{x}}=\left[\begin{array}{ccc}
-\mathbf{K}_{D} & -\mathbf{K}_{P} & -\mathbf{K}_{I} \\
\mathbf{I} & \boldsymbol{r}(t) \mathbf{S} & \mathbf{0} \\
\mathbf{0} & \mathbf{I} & \mathbf{0}
\end{array}\right] \mathbf{x} \triangleq \mathbf{A}(t) \mathbf{x}
$$

which is linear and non-autonomous system, since the yaw rate $r(t)$ depends on $t$. The equilibrium point is the origin, which yields $\left(\tilde{\mathbf{v}}_{0}, \mathbf{e}_{0}, \mathbf{z}_{0}\right)=\left(\mathbf{0}, \mathbf{0},-\mathbf{K}_{l}^{-1} \mathbf{T} \mathbf{M}^{-1} \mathbf{b}\right)$.

Theorem 2. The equilibrium point is GES.

Proof. Write the system $\dot{\mathbf{x}}=\mathbf{A}(t) \mathbf{x}$ as

$$
\begin{aligned}
& \dot{\mathbf{x}}=\mathbf{A}_{0} \mathbf{x}+\mathbf{B} \xi \\
& \mathbf{y}=\mathbf{C x} \\
& \xi=r(t) \mathbf{y}
\end{aligned}
$$


All components of the vectors $r_{\max } \mathbf{y}(t)-\xi(t)$ and $r_{\max } \mathbf{y}(t)+\xi(t)$ are positive, and, in particular,

$$
\left(r_{\max } \mathbf{y}(t)-\xi(t)\right)^{T}\left(r_{\max } \mathbf{y}(t)+\xi(t)\right) \geq 0
$$

which yields

$$
r_{\max }^{2} \mathbf{y}^{T} \mathbf{y}-\boldsymbol{\xi}^{T} \boldsymbol{\xi} \geq 0
$$

Therefor the matrix II defined in Megretski and Rantzer (1997) has the form

$$
\mathrm{II}=\left[\begin{array}{cc}
r_{\max }^{2} \mathbf{I} & 0 \\
0 & -\mathbf{I}
\end{array}\right]
$$

and

$$
\left[\begin{array}{l}
\mathbf{y} \\
\xi
\end{array}\right]^{*}\left[\begin{array}{l}
\mathbf{y} \\
\xi
\end{array}\right] \geq 0
$$

Let $\tilde{\mathbf{y}}=\mathbf{C}\left(j \omega \mathbf{L}-\mathbf{A}_{0}\right)^{-1} \mathbf{B} \tilde{\boldsymbol{\xi}}=\mathbf{G}(j \omega) \tilde{\xi}$, then stability of the interconnected system is obtained if

$$
\begin{aligned}
{\left[\begin{array}{c}
\mathbf{G}(j \omega) \\
\mathbf{I}
\end{array}\right]^{*} \mathrm{II}\left[\begin{array}{c}
\mathbf{G}(j \omega) \\
\mathbf{I}
\end{array}\right] } & =\tilde{\boldsymbol{\zeta}}^{*}\left(r_{\max }^{2} \mathbf{G}^{*}(j \omega) \mathbf{G}(j \omega)-\mathbf{I}\right) \tilde{\xi} \\
& \leq-\varepsilon_{0} \tilde{\xi}^{*} \tilde{\xi} \leq 0, \varepsilon_{0}>0 \text { and } \forall \omega \in \mathbf{R}^{\prime}
\end{aligned}
$$

or that the matrix $r_{\max }^{2} \mathbf{G}^{*}(j \omega) \mathbf{G}(j \omega)$ - I is nonpositive $\forall \omega \in \mathbf{R}^{1}$. If it is so, and since $\mathbf{A}_{0}$ is Hurwitz there exist a positive definite matrix $\mathbf{P}=\mathbf{P}^{T}$ such that the Lyapunov function

$$
V(\mathbf{x})=\mathbf{x}^{T} \mathbf{P} \mathbf{x}
$$

has the time derivative along the trajectory given by

$$
\dot{V}=\mathbf{x}^{T} \mathbf{P}\left(\mathbf{A}_{0} \mathbf{x}+\mathbf{B} \xi\right)+\left(\mathbf{A}_{0} \mathbf{x}+\mathbf{B} \xi\right)^{T} \mathbf{P} \mathbf{x} \leq-\varepsilon_{1}\left(\mathbf{x}^{T} \mathbf{x}+\xi^{T} \xi\right) \leq 0, \varepsilon_{1}>0
$$

for $\forall x, \xi$ such that $r_{\max }^{2} \mathbf{x}^{T} C^{T} C \mathbf{x}-\xi^{T} \xi \geq 0$. Rewritten in matrix form

$$
\begin{aligned}
\dot{V} & =\left[\begin{array}{ll}
\mathbf{x}^{T} & \xi^{T}
\end{array}\right]\left[\begin{array}{cc}
\mathbf{P} \mathbf{A}_{0}+\mathbf{A}_{0}^{T} \mathbf{P}+r_{\max }^{2} \mathbf{C}^{T} \mathbf{C} & \mathbf{P B} \\
\mathbf{B}^{T} \mathbf{P} & -\mathbf{I}
\end{array}\right]\left[\begin{array}{l}
\mathbf{x} \\
\boldsymbol{\xi}
\end{array}\right] \\
& =\left[\begin{array}{ll}
\mathbf{x}^{T} & \boldsymbol{\xi}^{T}
\end{array}\right] \tilde{\mathbf{Q}}\left[\begin{array}{l}
\mathbf{x} \\
\xi
\end{array}\right] \leq \mathbf{0}
\end{aligned}
$$

the inequality (9) can be solved by LMI-methods, see Megretski and Rantzer (1997). The solution can then be written as

$$
\begin{aligned}
\dot{V} & =\mathbf{x}^{T}\left[\begin{array}{rl}
\mathbf{I} & \left.r(t) \mathbf{C}^{T}\right]
\end{array}\right]\left[\begin{array}{cc}
\mathbf{P} \mathbf{A}_{0}+\mathbf{A}_{0}^{T} \mathbf{P}+r_{\max }^{2} \mathbf{C}^{T} \mathbf{C} & \mathbf{P B} \\
\mathbf{B}^{T} \mathbf{P} & -\mathbf{I}
\end{array}\right]\left[\begin{array}{c}
\mathbf{I} \\
r(t) \mathbf{C}
\end{array}\right] \mathbf{x} \\
& =\mathbf{x}^{T}\left(\mathbf{P} \mathbf{A}_{0}+\mathbf{A}_{0}^{T} \mathbf{P}+r_{\max }^{2} \mathbf{C}^{T} \mathbf{C}+2 r(t) \mathbf{P B C}-r^{2}(t) \mathbf{C}^{T} \mathbf{C}\right) \mathbf{x} \\
& =-\mathbf{x}^{T} \mathbf{Q}(t) \mathbf{x}<0
\end{aligned}
$$

Exponential stability follows from the following: $\dot{V}(\mathbf{x}) \leq-\delta_{1} V(\mathbf{x})$, where $\delta_{1}=$ $\lambda_{\min }(\mathbf{Q}) / \lambda_{\max }(\mathbf{P})$, which yields

$$
V(\mathbf{x}(t)) \leq e^{-\delta_{1} t} V(\mathbf{x}(0))
$$




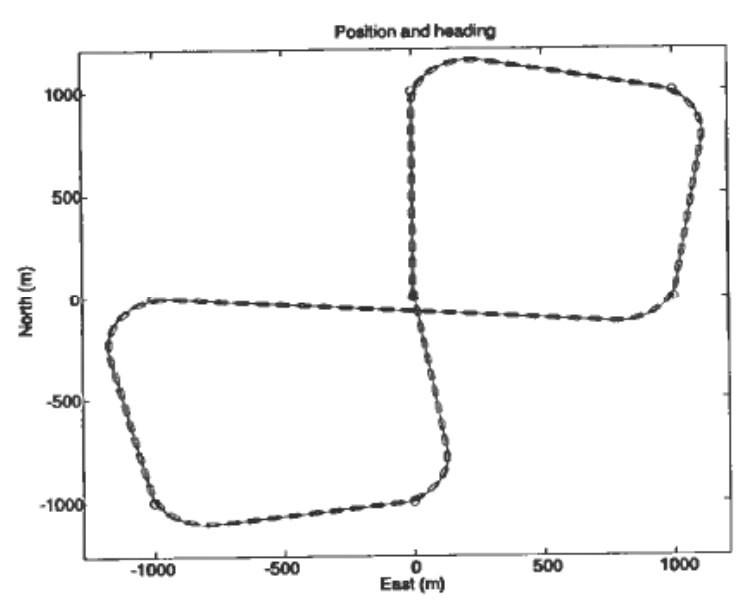

Figure 2. Simulated desired and actual position and heading through 7 way-points.
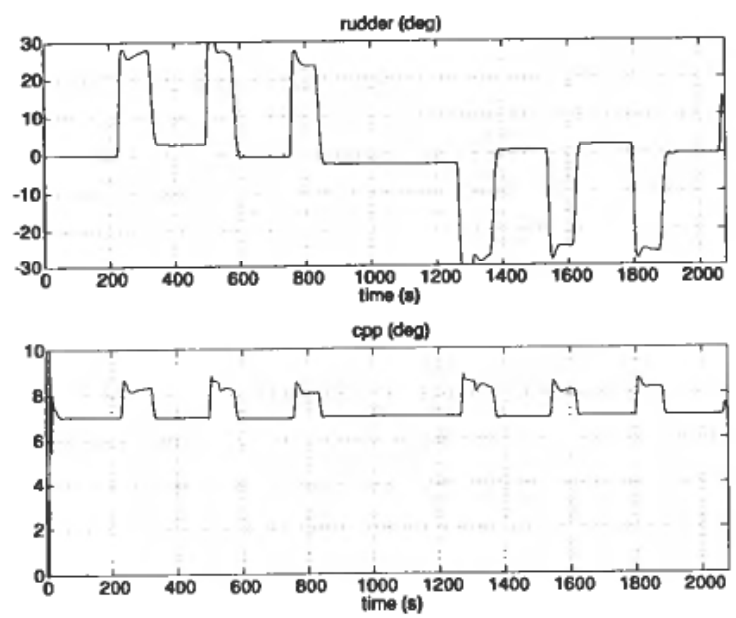

Figure 3. Rudder angle and controller pitch ratio for the propeller.
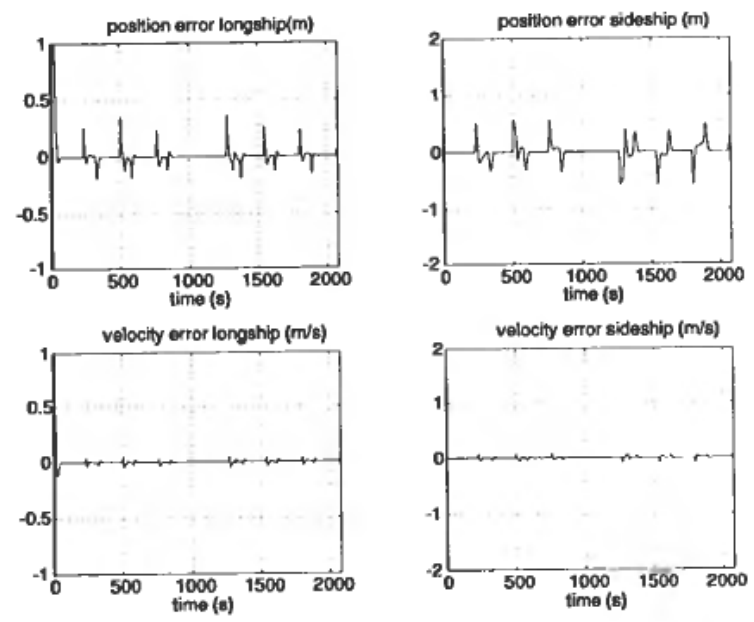

Figure 4. Cross-track and velocity errors. Between the way-points the errors converge to zero. 

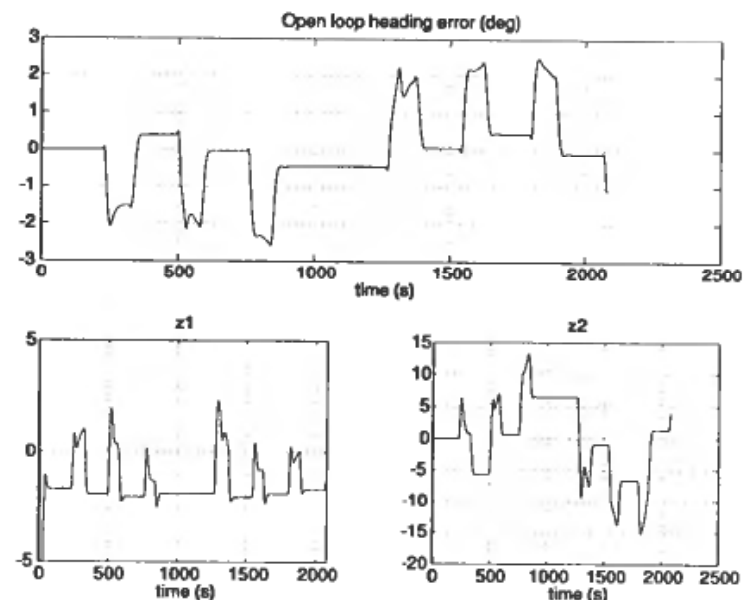

Figure 5. Upper plot shows that the heading error (open loop) is within $\pm 1^{\circ}$ and corresponding integrated longship and cross-track errors are bounded.

\section{Reference Generator}

The reference trajectory is generated by using a 1st order Nomoto model of the ship. That is,

$$
\dot{r}_{r}=-\frac{1}{T} r_{r}+\frac{K}{T} \Delta \psi_{r}
$$

where $T$ and $K$ are the Nomoto parameters for the simulated ship. $\Delta \psi_{r}=\psi_{d}-\psi_{r}$ is the heading error between the desired heading and the reference heading. A new way-point is selected when the distance from the ship to the way-point is less than 2 times the ship's length. The reference yaw rate is limited to $\left|r_{r}\right| \leqslant 1 \% s$ and the desired heading angle $\psi_{d}$ is computed by using the LOS method (Fossen 1994).

$$
\psi_{d}(t)=\operatorname{atan} 2\left(y_{w}-y_{r}(t), x_{w}-x_{r}(t)\right)
$$

where atan2 is the four quadrant arctangent function. $\mathbf{p}_{w}=\left[x_{w}, y_{w}\right]^{T}$ is the next way-point and $\mathbf{p}_{r}=\left[x_{r}(t), y_{r}(t)\right]^{T}$ is the reference position. The desired surge and sway velocities $u_{d}, v_{d}$ are assumed to be constant, that is $\mathbf{v}_{d}=\left[u_{d}, v_{d}\right]^{T}=\left[U_{d}, 0\right]^{T}$ where $U_{d}$ is the desired forward speed. The update laws are

$$
\begin{aligned}
& \dot{u}_{r}=K_{11}\left(u_{d}-u_{r}\right) \\
& \dot{v}_{r}=K_{22}\left(v_{d}-v_{r}\right) \\
& \dot{r}_{r}=-\frac{1}{T} r_{r}+\frac{K_{33}}{T} \Delta \psi_{r}
\end{aligned}
$$

The reference position is then found from $\dot{\eta}_{r}=\mathbf{R}_{r} \boldsymbol{\nu}_{r}$, where the rotation matrix $\mathbf{R}_{r}$ is given by

$$
\mathbf{R}_{r}=\mathbf{R}_{r}\left(\psi_{r}\right)=\left[\begin{array}{ccc}
\cos \psi_{r} & -\sin \psi_{r} & 0 \\
\sin \psi_{r} & \cos \psi_{r} & 0 \\
0 & 0 & 1
\end{array}\right]
$$

and the desired position at the $V R P$ is evaluated from

$$
\boldsymbol{\eta}_{d}=\boldsymbol{\eta}_{r}+\mathbf{R}_{r} \mathbf{d}_{r}
$$


where $\mathbf{d}_{r}=\left[x_{v}, y_{v}, 0\right]^{T}$ is the vector from the center of rotation to the VRP. Higher order derivatives $\boldsymbol{\eta}_{d}$ is found by using a low-pass filter.

\section{Simulation Study}

A simulation study is performed for the Japanese training ship Shiojimaru. The desired path was generated with the following parameters: $K_{11}=K_{22}=1 \cdot 0, K_{33}=0.1$ and $T=1.6 \mathrm{~s}$. The yaw rate is limited to $1 \% \mathrm{~s}$. The proposed control law given by (21) is used to control the ship through 7 way-points. The forward speed of the vessel is controlled by the stern propeller. The propeller revolution is kept constant and the CPP is used to control the thrust force. A rudder is used to control the sway and yaw motions according to the thrust model given by (13). The selected forward speed is $U_{d}=4.0 \mathrm{~m} / \mathrm{s}$, and the way-points $\mathbf{p}_{w}$ are selected as $\mathbf{p}_{w}=\{(0,0),(1000,0),(1000,1000)$, $(0,1000),(0,-1000),(-1000,-1000),(-1000,0),(0,0)\}$. Wind forces corresponding to $U_{W}=5 \mathrm{~m} / \mathrm{s}$ from North were also added. The controller gains are chosen as $\mathbf{K}_{P}=\operatorname{diag}(0 \cdot 1,0 \cdot 5), \mathbf{K}_{D}=\operatorname{diag}(0.5,2 \cdot 0), \mathbf{K}_{I}=\operatorname{diag}(0 \cdot 01,0.05)$ and the $V R P$ is given by $\mathbf{d}_{v}=[40 \cdot 0,0]^{T}$. Simulation results are presented in Figures 2-5. As seen from Figure 5, the open loop heading error is within $\pm 1^{\circ}$ for this disturbance and speed.

\section{Conclusions}

A nonlinear way-point tracking controller for ships has been presented and stability has been proved by using Lyapunov theory. A nonlincar model of the Japanese training ship Shiojimaru was used for computer simulation. Both the position and velocity error converges exponentially to zero. Also wind forces from the North were added to verify the performance and the robustness of the proposed controller.

\section{Appendix A. Mathematical Model of Shiojimaru}

The nonlinear model of Shiojimaru (Ohtsu et al. 1996), is written:

$$
\mathbf{M} \dot{\boldsymbol{\nu}}+\mathbf{n}(\boldsymbol{\nu})=\boldsymbol{\tau}
$$

where $\boldsymbol{\nu}=[u, v, r]^{T}$ and $\mathbf{M}=\operatorname{diag}\left(m+m_{x}, m+m_{y}, I_{z z}+J_{z z}\right)$. Here $m$ is mass of ship, $m_{x}$, $m_{y}$ is added mass in $x$ - and $y$-direction. $I_{z z}+J_{z z}$ is inertial and added inertial moment around the $z$-axis. The nonlinear vector $\mathbf{n}(\boldsymbol{\nu})$ represents the Coriolis, centripetal and the hydrodynamic forces:

$$
\begin{aligned}
& n_{1}=C_{l}|u| u-X_{v} V|v|-X_{v r} v r-X_{r} V|r|-X_{v v} v^{2}-X_{r r} r^{2} \\
& n_{2}=-Y_{v} V v-Y_{v v}|v| v-Y_{r} V r-Y_{r r}|r| r-Y_{v r}|r| v \\
& n_{3}=-N_{v} V v-N_{v v}|v| v-N_{r} V r-N_{r r}|r| r-N_{v r}|r| v
\end{aligned}
$$

where $V$ is the ship's speed $V=\sqrt{u^{2}+v^{2}}$. The vector $\tau$ represents all external forces due to the propeller, rudder and environmental disturbances.

$$
\begin{aligned}
& \tau_{1}=X_{P}+X_{R}+X_{W} \\
& \tau_{2}=Y_{R}+Y_{W} \\
& \tau_{3}=N_{R}+N_{W}
\end{aligned}
$$

Here the subscripts $P, R$, and $W$ denote the forces and moment corresponding to the 
propeller, rudder and disturbances. The thrust force of the propeller (constant pitch angle $\theta_{P}$ ) is:

$$
\begin{aligned}
X_{P}= & (1-t) \rho n^{2} D_{P}^{4}\left(C_{0}+C_{1} \theta_{P}+C_{2} J_{P}\right. \\
& +C_{3} \theta_{P} J_{P}+C_{4} \theta_{P}^{2}+C_{5} J_{P}^{2}+C_{6} \theta_{P}^{2} J_{P} \\
& \left.+C_{7} \theta_{P} J_{P}^{2}+C_{8} \theta_{P}^{3}+C_{9} J_{P}^{3}\right)
\end{aligned}
$$

Here $t$ denotes the thrust deduction fraction and $n, D_{P}$ and $J_{P}$ are the propeller revolutions, propeller diameter and advance coefficient, respectively. $C_{1} \sim C_{9}$ are empirical coefficients. The rudder forces are written:

$$
\begin{aligned}
X_{R} & =-\left(1-t_{R}\right) F_{N} \sin \delta \\
Y_{R} & =-\left(1-a_{H}\right) F_{N} \cos \delta \\
N_{R} & =-\left(x_{R}+a_{H} x_{H}\right) F_{N} \cos \delta
\end{aligned}
$$

where $t_{R}, a_{H}$ and $x_{H}$ denote empirical coefficients due to hull-propeller interactions and $x_{R}$ is the rudder position. The rudder normal force $F_{N}$ is

$$
F_{N}=\frac{1}{2} \rho A_{R} f_{\alpha}\left(U_{R}^{2} \sin \delta+\gamma_{R}\left(v+l_{R} r\right) U_{R} \cos \delta\right)
$$

where $A_{R}$ and $f_{x}$ denote the projected rudder area and its normal force coefficient. $\gamma_{R}$, $l_{R}$ are empirical coefficients representing the fairing effects of the stream behind the hull. The effective rudder inflow is:

$$
U_{R}=\left(\varepsilon-k_{x}\right)\left(1-w_{P}\right) u+k_{x}\left(0 \cdot 7 \pi D_{P} n\right) \tan \theta_{P}
$$

where $\varepsilon$ denotes the ratio of axial velocity between the propeller position and the rudder position. $k_{x}$ denotes the propeller acceleration fraction. The rudder dynamics is modeled by

$$
\dot{\delta}=\frac{\left(\delta_{c}-\delta\right)}{\left(\left|\delta_{c}-\delta\right| T_{R U D}+a\right)}
$$

where $\delta_{c}$ is the rudder command and $T_{R U D}$ is the rudder time constant and $a$ is a constant. The rudder angle is limited to $\delta_{\min } \leq \delta \leq \delta_{\max }$. The wind forces on the ship's superstructure can be represented as follows (Fossen 1994):

$$
\begin{aligned}
Y_{W} & =\frac{1}{2} \rho_{A} A_{o f} U_{W}^{2} C_{X} \\
Y_{W} & =\frac{1}{2} \rho_{A} A_{o s} U_{W}^{2} C_{Y} \\
N_{W} & =\frac{1}{2} \rho_{A} A_{o s} L_{P P} U_{W}^{2} C_{N}
\end{aligned}
$$

where $C_{X}, C_{Y}$ and $C_{N}$ are the wind coefficients. $\rho_{A}$ is the density of air. $A_{o f}$ and $A_{o s}$ are the lateral and transverse projected area of the superstructure respectively. The coefficients $C_{X}, C_{Y}$ and $C_{N}$ are all functions of the wind direction relative to the ship. An approximation is:

$$
\begin{aligned}
& C_{X}=\hat{C}_{X} \cos \alpha \\
& C_{Y}=\hat{C}_{Y} \sin \alpha \\
& C_{N}=\hat{C}_{N} \sin 2 \alpha
\end{aligned}
$$

where $\hat{C}_{X}, \hat{C}_{Y}$ and $\hat{C}_{N}$ are constants. 


\section{Appendix B. Zero Dynamics}

Since we do not control the yaw mode directly, we must ensure that the ship will not rotate $180^{\circ}$ as discussed in the introduction. The zero dynamics at the equilibrium point $\left(\tilde{\mathbf{v}}_{0}, \mathbf{e}_{0}, \mathbf{z}_{0}\right)$ is found by applying the control law (21) together with (12):

$$
\dot{\boldsymbol{\nu}}=\mathbf{E} \dot{\mathbf{v}}_{d}+\mathbf{F}[\mathbf{n}(\boldsymbol{\nu})-\mathbf{b}]
$$

where

$$
\begin{aligned}
& \mathbf{E}=\left\{e_{i j}\right\}=\mathbf{M}^{-1} \mathbf{B B}_{u}^{-1} \\
& \mathbf{F}=\left\{f_{i j}\right\}=\mathbf{M}^{-1}\left(\mathbf{B B}_{u}^{-1} \mathbf{T} \mathbf{M}^{-1}-\mathbf{I}\right)
\end{aligned}
$$

Based on the model in Appendix A, the closed-loop dynamics can be written as:

$$
\begin{aligned}
& \dot{u}=\dot{u}_{d} \\
& \dot{v}=e_{22} \dot{v}_{d}-x_{a}\left(f_{32} n_{2}+f_{33} n_{3}\right) \\
& \dot{r}=e_{32} \dot{v}_{d}+f_{32} n_{2}+f_{33} n_{3}
\end{aligned}
$$

where we assumed $\mathbf{d}_{v}=\left[x_{v}, 0\right]^{T}, \mathbf{b}=\mathbf{0}$ and $\tilde{\mathbf{R}}=\mathbf{I}$. Notice, that the zero dynamics does not depend on the selected controller gain since the error dynamics is zero at the equilibrium point. Further, we see that (B.4) yields exact tracking in surge, whereas (B.5) and (B.6) yield the zero dynamics for sway and yaw. Let the desired reference be zero, $v_{d}=0$, then the yaw $r$ rate will be proportional to the sway velocity $v$ according to

$$
r=-v / x_{v}
$$

since $v_{d}-v-x_{v} r=0$ at the equilibrium point. The physical interpretation of the constraint (B.7) is that the ship's center of rotation is allowed to move on a circle, where $x_{v}$ is the distance from the center of the rotation to the VRP. The coupled sway/yaw closed-loop stability can be evaluated by requiring $r=-v / x_{v}$ in (B.5)-(B.6). Note, that

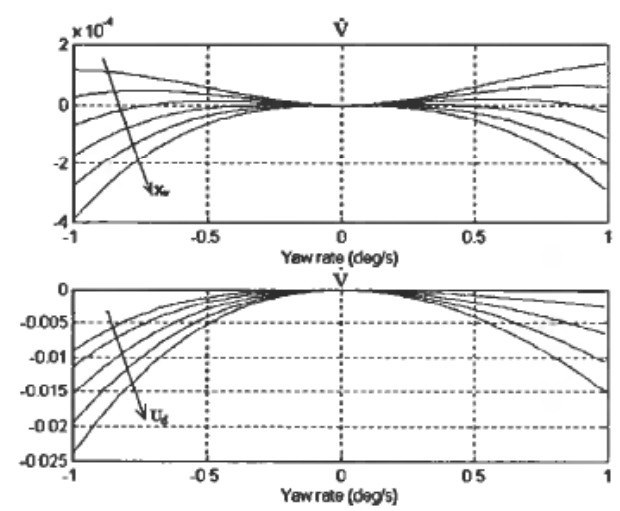

Figure B.1. Upper plot: $\dot{V}$ for $U_{d}=4.0 \mathrm{~m} / \mathrm{s}$ and $x_{v}=\{5,6,7,8,9,10\} \mathrm{m}$. Lower plot: $\dot{V}$ for $x_{\mathrm{r}}=40.0 \mathrm{~m}$ and $U_{d}=\{0,1,2,3,4,5\} \mathrm{m} / \mathrm{s}$. 
we only have to consider one degree of freedom, since both the sway and yaw motions are coupled by (B.7). From Appendix A we find

$$
\begin{aligned}
& n_{2}=Y_{v} U_{d} x_{v} r+Y_{v v}\left|x_{v} r\right| x_{v} r-Y_{r} U_{d} r-Y_{r r}|r| r+Y_{v}|r| x_{v} r \\
& n_{3}=N_{v} U_{d} x_{v} r+N_{v v}\left|x_{v} r\right| x_{v} r-N_{r} U_{d} r-N_{r r}|r| r+N_{v}|r| x_{v} r
\end{aligned}
$$

Define $V=\frac{1}{2} r^{2}$. The yaw/sway motion will be stable if

$$
\dot{V}=r \dot{r}<0, \quad \forall r \neq 0
$$

As seen from (B.8) and (B.9), $\dot{V}$ is a function of both the forward speed $U_{d}$ and $x_{v}$.

In Figure B.1, the upper plot shows the $\dot{V}$ for $x_{v}=\{5,6,7,8,9,10\} m$ and $U_{d}=4.0 \mathrm{~m} / \mathrm{s}$. Notice, that the choice of $V R P$ is critical for the closed-loop stability. For $x_{v}<8 \mathrm{~m}$, the sway and yaw motion is unstable, e.g., the ship will rotate around. The lower plot shows $\dot{V}$ for different forward speeds for $x_{v}=40.0 \mathrm{~m}$ and we notice that the ship will be stable for all speeds. $\dot{V}$ becomes more negative for higher speed which is reasonable since the hydrodynamics forces will be more significant. Since $V>0$ and $\dot{V}<0 \forall r \neq 0$, the yaw motion is asymptotically stable for constant set-points.

\section{REFERENCES}

Fossen, T. I. (1994). Guidance and Control of Ocean Vehicles, John Wiley and Sons Ltd.

Fossen, T. I. and StRAND, J. P. (1999). Passive nonlinear observer design for ships using Lyapunov methods: experimental results with a supply vessel. Automatica.

FosSEN, T. I., GODHAVN, J.-M., BERGE, S. P. and LiNDEGAARD, K.-P. (1998). Nonlinear control of underactuated ships with forward speed compensation. Proc. of the IFAC NOLCOS'98.

GoDHAVN, J.-M. (1997). Topics in Nonlinear Motion Control: Nonholonomic, Underactuated and Hybrid Systems. PhD thesis. Dep. of Eng. Cybernetics, NTNU, Trondheim.

GodHAVN, J.-M., Fossen, T. I. and BerGE, S. P. (1998). Nonlinear and adaptive backstepping designs for tracking control of ships. International Journal of Adaptive Control and Signal Processing.

HolzHÜTER, T. and SCHULTZE, R. (1996). Operating experience with a high-precision track controller for commercial ships. Control Eng. Practice, CEP-4(3), 343-350.

LINDEGAARD, K.P. (1997). Nonlinear tracking control and station-keeping of underactuated ships. Master's thesis. Dep. of Eng. Cybernetics, NTNU, Trondheim.

MEGRETSKI, A. and RANTZER, A. (1997). System analysis via integral quadratic constraints. IEEE Trans. on Control System Technology, TCST-42(6), 93-106.

OHTSU, K., SHOI, K. and OKAZAKI, T. (1996). Minimum-time maneuvering of a ship, with wind disturbances. Control Eng. Practice, CEP-4(3), 385-392. 